\title{
Corrections
}

\section{Correction: Mashiko et al., Comparative Anatomy of Marmoset and Mouse Cortex from Genomic Expression}

In the article "Comparative Anatomy of Marmoset and Mouse Cortex from Genomic Expression" by Hiromi Mashiko, Aya C. Yoshida, Satomi S. Kikuchi, Kimie Niimi, Eiki Takahashi, Jun Aruga, Hideyuki Okano, and Tomomi Shimogori, which appeared on pages 5039-5053 of the April 11, 2012 issue, one gene was incorrectly identified. The annotation for the GenBank marmoset mRNA sequence record XM-002751158 currently contains an error, incorrectly identifying the mRNA as the product of the ntng2 gene, while correctly indicating that the encoded protein is netrin-G1. Netrin-G1 is the product of the ntng1 gene. As a result, each occurrence of "Ntng2" in the text, figure legends, and tables should be replaced with "Ntng1," and the corresponding encoding protein product listed in Table 1 should be "Netrin G1" instead of "Netrin G2." This correction has no substantial consequence for the major conclusions or the discussion of the results.

DOI: 10.1523/JNEUROSCI.5030-12.2012 